\title{
POD Spectrum of the Wake behind a Circular Cylinder
}

\author{
Václav Uruba ${ }^{1,2, *}$, and Pavel Procházka ${ }^{1}$ \\ ${ }^{1}$ Department of Fluid Dynamics, Institute of Thermomechanics of the Czech Academy of Sciences, \\ Dolejškova 5, 182 00, Czech Republic \\ ${ }^{2}$ Department of Power System Engineering, Faculty of Mechanical Engineering, University of West \\ Bohemia, Universitní 8, 30614 Plzeň, Czech Republic
}

\begin{abstract}
The wake dynamics behind a long circular cylinder in crossflow was studied using the POD method. The temporal parts of POD modes, Chronoses, are subjected to frequency analysis. Five groups of modes are distinguished according to the frequency contents. The low order high energy modes contain the vortex shedding frequency or its harmonics up to $3^{\text {rd }}$ order plus Kolmogorov spectrum. The higher order modes are characterized by combination of Kolmogorov spectrum with the white noise spectrum, its importance grows with the mode order. The very high order modes are characterised by the white noise spectrum only.
\end{abstract}

\section{Introduction}

The wake behind a long circular cylinder, virtually infinite, belongs to so called canonical cases in fluid mechanics, those what are well investigated by all means available. Many experimental studies and mathematical simulations could be find in literature (see e.g. [1, 8, 9]).

The wake dynamical behaviour is presented to be governed by the periodical phenomenon characterized by fundamental frequency. This phenomenon is connected with periodical separation of vortical structures, so called von Kármán-Bénard vortex street. The dimensionless frequency is called Strouhal number St and it is defined as follows: $\mathrm{St}=(f . d) / U$, where $f$ is the fundamental frequency, $d$ is the cylinder diameter and $U$ is the incoming velocity. Typical value of the Strouhal number is about $\mathrm{St}=0.2$ in the broad range of Reynolds numbers, higher than 200 (see e.g. [1]).

The dynamics of the cylinder wake is considered to be turbulent for those Reynolds numbers (see e.g. [3, 4]) with a single predominant frequency peak in the spectrum. However, the flowing fluid represents itself strongly non-linear dynamical system, this fact could be demonstrated by Navier-Stokes equations, as the mathematical model. From the theory of non-linear dynamical systems it is known that the higher harmonics components should appear in the system dynamics as a rule. However presence of higher harmonics in the wake have not been studied yet.

\footnotetext{
* Corresponding author: uruba@it.cas.cz
} 
The presented study brings experimental evidence of higher harmonics presence in the wake flow-field dynamics. Moreover, the dense spectrum is detected confirming the welldeveloped turbulence existence in the wake.

\section{Proper Orthogonal Decomposition}

The Proper Orthogonal Decomposition (hereinafter POD) becomes in a classical tool to study dynamics of extended dynamical systems. The POD provides an unbiased technique for identifying deterministic features from a random, fine grained turbulent flow. The method extracts the candidate which is best correlated, in a statistical sense, with the background velocity field. A set of structures is identified with the orthogonal eigenfunctions of the decomposition theorem of probability theory. This is thus a systematic way to find organized motions in a given set of realizations of a random field.

The goal of the decomposition methods in general is to look for a specific base with a distinct physical meaning. In the case of the POD that means an orthonormal basis corresponding to decorrelated (i.e. orthogonal) modes maximizing the spatio-temporal data variance. In the case of application on velocity field the maximized quantity physical interpretation is a total turbulent kinetic energy.

In this paper we will consider the POD in its extended version, which uses decomposition in both spatial and temporal domains. This version is also called $\mathrm{Bi}$ Orthogonal Decomposition, see e.g. [5].

Using the POD approach the dynamical behaviour of the flow-field is represented by linear combination of the POD modes. Number of modes is equal to the number of snapshots or number of degree of freedom, whatever is lower. Each POD mode consists of three parts: amplitude, spatial distribution (Topos) and distribution in time (Chronos). Mathematical representation is as follows:

$$
\mathbf{u}(\mathbf{x}, t)=\sum_{k} a_{k} \overline{\boldsymbol{\varphi}_{k}(\mathbf{x})} \boldsymbol{\Psi}_{k}(t),
$$

the bar denotes complex conjugate (however all functions are typically real), $\boldsymbol{\varphi}_{k}(\mathbf{x})$ are spatial eigenfunctions Toposes, $\boldsymbol{\Psi}_{k}(t)$ are temporal eigenfunctions Chronoses, $a_{k}$ are amplitudes. Then, $a_{k}^{2}$ are the common eigenvalues. Both Toposes and Chronoses are normalized to form orthonormal bases, so they are dimensionless, the amplitude has physical dimension of the velocity, i.e. $[\mathrm{m} / \mathrm{s}]$. Please note that the eigenvalue, the amplitude square, corresponds to the mode turbulence kinetic energy multiplied by an arbitrary constant.

To compare the energies of the individual modes in time and frequency space, we will use the product of amplitude and Chronos: $a_{k} \boldsymbol{\psi}_{k}(t)$, see [6].

\section{Experimental Setup and Instrumentation}

The experimental setup and the experimental method used will be specified.

\subsection{Experimental Setup}

The cylinder of diameter $15 \mathrm{~mm}$ is placed perpendicularly to the flow in the test-section inlet of the blown-down facility. The cross-section of the closed test-section is $250 \times 250 \mathrm{~mm}^{2}$ in the position of the cylinder. The velocity on the test-section inlet was $U_{\mathrm{i}}=5 \mathrm{~m} / \mathrm{s}$ corresponding to the Reynolds number about 4815 . 
The situation is depicted schematically in Figure 1, there is the cylinder, Cartesian coordinate system with origin located on the cylinder axis and plane of measurement (in green).

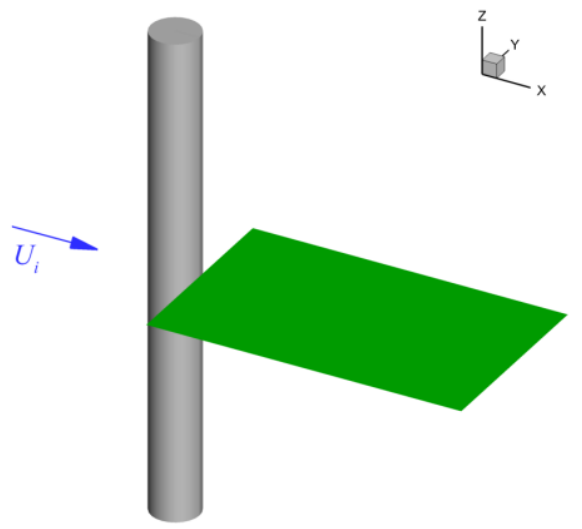

Fig. 1. Schematics of experimental setup.

The plane of measurement is located in the middle of the test section height. Blockage of the test section cross-section was about $6 \%$.

\subsection{Instrumentation}

The velocity vector field was evaluated using Particle Image Velocimetry (PIV) method.

The measurement apparatus consists of laser and CMOS camera by Dantec company. The laser is New Wave Pegasus, Nd:YLF double head with wavelength of $527 \mathrm{~nm}$, maximal frequency $10 \mathrm{kHz}$, shot energy is $10 \mathrm{~mJ}$ (for $1 \mathrm{kHz}$ ) and corresponding power is $10 \mathrm{~W}$ per one head. The camera Phantom V611 with resolution of 1280 x 800 pixels is able to acquire double snaps with frequency up to $3000 \mathrm{~Hz}$ (full resolution) and it uses internal memory 8 GB. The data were acquired and post-processed in DynamicStudio software.

The PIV measurement was performed in the plane perpendicular to the cylinder axis and parallel to the flow direction $(x y)$, shown in green in Fig. 1. The evaluated velocity fields consisting of $159 \times 99$ vectors were acquired in frequency $2 \mathrm{kHz}$, one record contained 4000 double-snapshots representing $2 \mathrm{~s}$ in real time.

The SAFEX particles, oil droplets $1 \mu \mathrm{m}$ in diameter, have been used.

More details could be found e.g. in $[2,7]$.

\section{Results}

The results are to be presented in dimensionless form, coordinates are expressed in multiples of the cylinder diameter $d$ and velocities in multiples of incoming velocity $U_{\mathrm{i}}$. All other quantities are non-dimensioned with help of the $\mathrm{d}$ and $U_{\mathrm{i}}$ as well.

\subsection{The Flow-Field}

The flow-field is to be shown in terms of time-mean quantities first.

In Figure 2 the time mean velocity vector field is shown. For the sake of clarity, the corresponding vector-lines in blue are added in an arbitrary manner. 


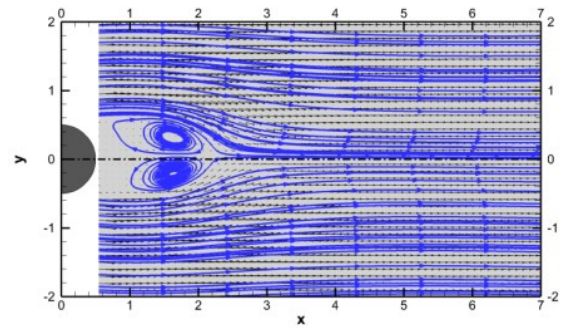

Fig. 2. Time mean velocity vector field with corresponding vector-lines.

The two recirculation zones are identified in the near-wake just behind the cylinder, ending in the distance 2 . However the size of the wake itself was much bigger, as could be seen from Figure 3, where the streamwise velocity component and Turbulent Kinetic Energy (TKE) distributions are shown.
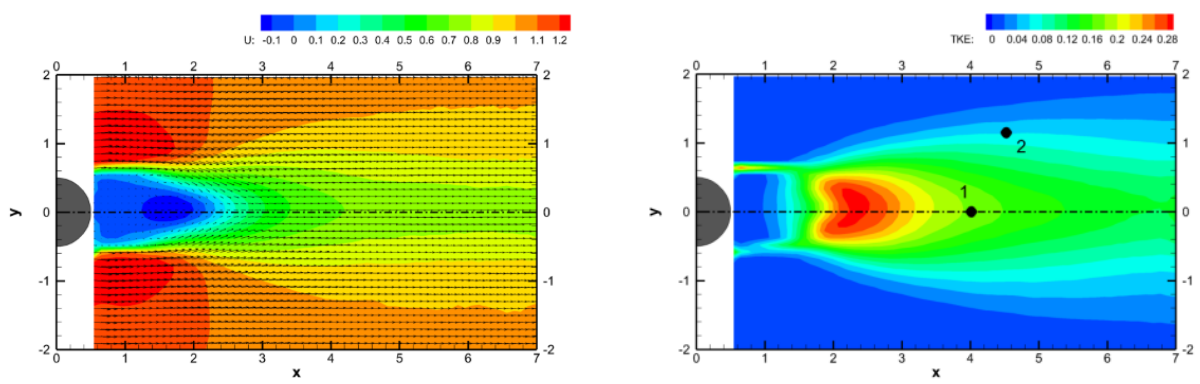

Fig. 3. Time mean fields of streamwise velocity component (left) and Turbulent Kinetic Energy (right).

In of streamwise velocity component distribution we could see the velocity overshoot on the near-wake sides around the points $[1 ;+-1]$, the velocity deficit region (in yellow) show the real wake extend. The same information could be gathered from the TKE distribution, where increasingly perturbed region is shown. The maximum fluctuating activity is near the axis in the distance about $x=2.5$ (red region).

The two points for spectra evaluation have been defined, the point 1 on the axis in position $[4 ; 0]$ and the point 2 close to the wake border in position $[4.53 ; 1.15]$.

In Figure 4 distribution of the $z$ mean vorticity component and its standard deviation are shown.
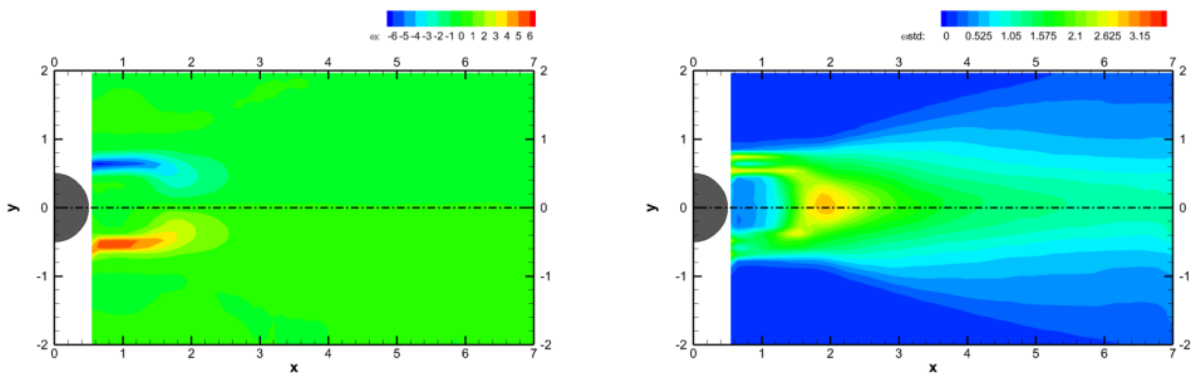

Fig. 4. Time mean fields of vorticity (left) and vorticity standard deviation (right). 
The vorticity is high positive (red) and negative (blue) within the shear layers behind the cylinder contour. The vorticity fluctuations are detected in the whole wake with maximum close to the axis in the distance 2 behind the cylinder.

The spectra of the streamwise and spanwise velocity components were evaluated in the points 1 and 2 for both streamwise and spanwise velocity components, see Figure 5. The standard method with smoothing using Hamming window was used.
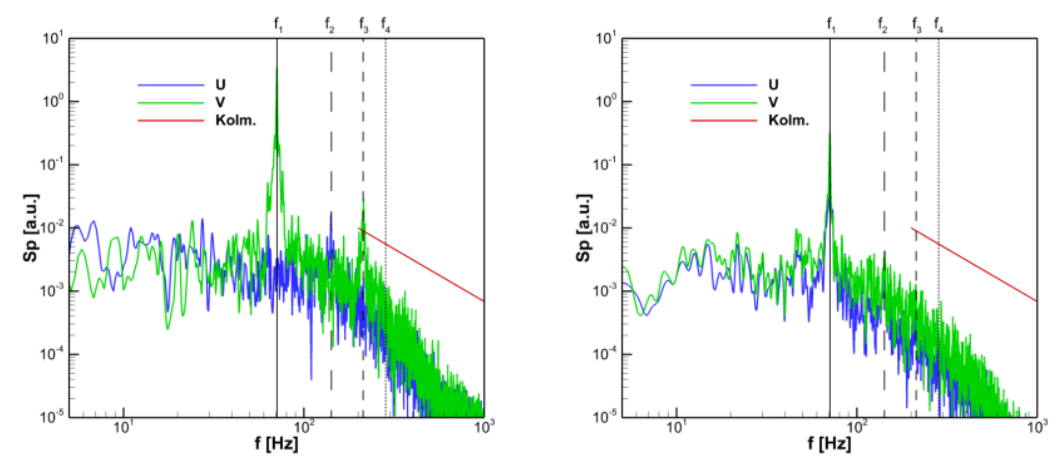

Fig. 5. Spectra of streamwise and spanwise velocity components in reference points 1 (left) and 2 (right).

The lines define the frequencies. The fundamental frequency $f_{1}=71 \mathrm{~Hz}$ corresponds to the Strouhal number $\mathrm{St}=0.21$ and it is depicted by the full line. The higher harmonics are shown by broken lines with decreasing pattern length: $f_{2}=142 \mathrm{~Hz}, f_{3}=213 \mathrm{~Hz}$ and $f_{4}=284 \mathrm{~Hz}$. The streamwise velocity component is blue, spanwise velocity component is in green and in red is shown spectrum after the Kolmogorov hypothesis in inertial region $(-5 / 3$ law).

Please note that the spectra in points 1 and 2 are considerably different. Although both spectra contain the turbulent part, which is typical by the continuous character obeying the Kolmogorov law in inertial region for frequencies $100 \mathrm{~Hz}$ and higher.

The spectrum of the streamwise velocity component $U$ in point 1 located on axis, exhibits a peak on the second harmonics, while on the fundamental harmonics there is no peak. In contrast, the spectrum of the spanwise velocity component $V$ shows two peaks, the highest on the fundamental frequency and the second, weaker, on the third harmonics.

In the point 2 close to the wake border, both spectra show distinct peak on fundamental frequency only.

\subsection{POD}

The result of the POD analysis are to be presented. The set of 4000 snapshots were decomposed using a standard POD procedure implemented in the DynamicStudio software. The 4000 POD modes have been evaluated.

In Figure 6 the kinetic energy distribution among the POD modes is shown. The first quantity the Energy Fraction (EF) express the percentage shear of a given POD mode on the total kinetic energy, the Accumulated Energy (AE) expresses the sum energy of all lower order POD modes and Residual Energy (RE) is an unexplained part of the total kinetic energy. 


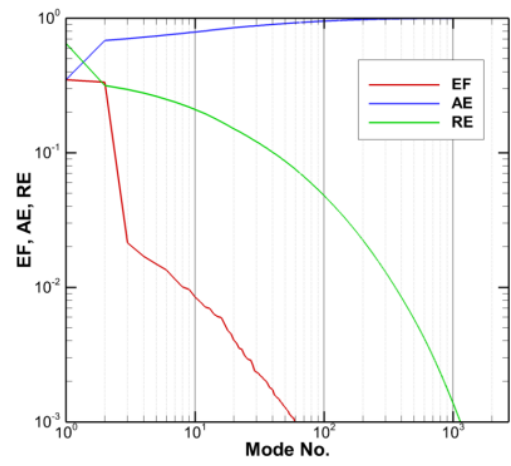

Fig. 6. Kinetic energy distribution among the POD modes.

The first two POD modes contain more than $68 \%$ of the total kinetic energy, the mode $135 \%$ (i.e. 0.35 ) and the mode $233 \%$. The higher modes are considerably less energetic nevertheless the first 10 POD modes cover about $78 \%$ of the accumulated kinetic energy (AE), thus the Residual Energy is $22 \%$. This result indicate, that there is a quasiperiodical process which is covered by the POD modes 1 and 2 .

The Toposes and Chronoses were evaluated for all 4000 POD modes. Spectra of all Chronoses have been evaluated as well, however the product Chronos and amplitude $a_{k} \boldsymbol{\psi}_{k}(t)$ was analysed for each mode, as explained in Chapter 2.

In Figure 7 the power-spectra for selected modes are shown, as well as the Kolmogorov law (the red line).

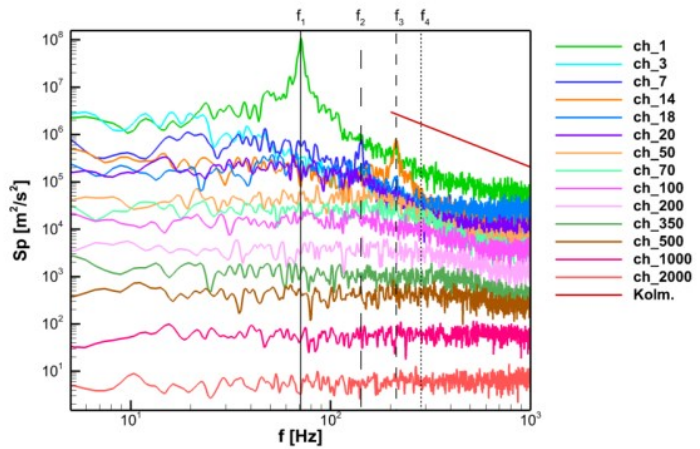

Fig. 7. Spectra of the selected Chronoses.

It is clear, that the energy of modes is decaying with the mode order, as the energy corresponds to the integral of each individual spectrum. The spectra have some common as well as some different features. Some of them obey the Kolmogorov $-5 / 3$ law (e.g. ch1). The others show the tendency to "white noise" this means the constant value along the frequencies (e.g. ch2000). Some of them contain peaks on the fundamental frequency or on higher harmonics.

In the following paragraph we will give an approximate qualitative overview of the POD dynamical features. The modes are sorted out into several groups. For the each presented POD mode the two pictures will be given. The spectrum of the Chronos on the left-hand side and the Topos topology on the right-hand side. The Topos is represented by vector-field and the vorticity distribution. The vorticity is evaluated from the in-plane 
velocity component and thus represents the component perpendicular to the plane of measurement. Red colour means positive, counter-clockwise orientation while negative, clockwise direction is depicted in blue.

Group 1: $f_{1}$ and Kolmogorov law.

This group of POD modes is characterized by the peak on fundamental frequency $f_{1}$ and by presence of the Kolmogorov law.

To this group belong the POD modes 1 and 2 only. In Figures 8 and 9 the POD modes 1 and 2 are shown.
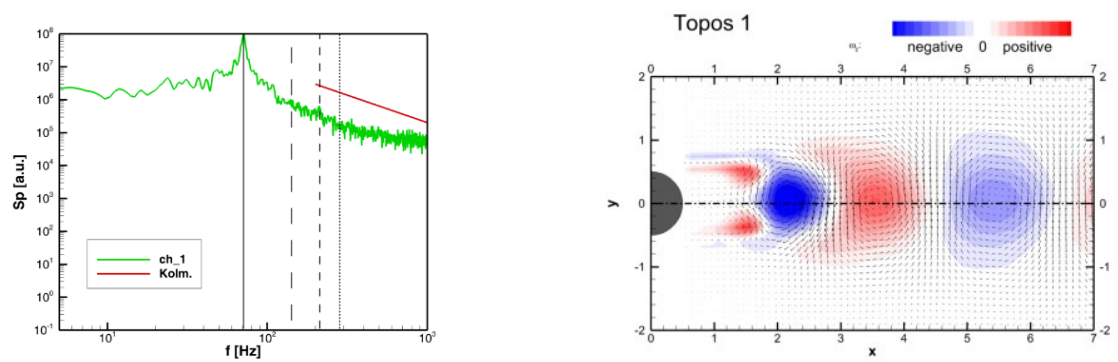

Fig. 8. POD mode 1, spectrum of Chronos (left) and Topos (right).
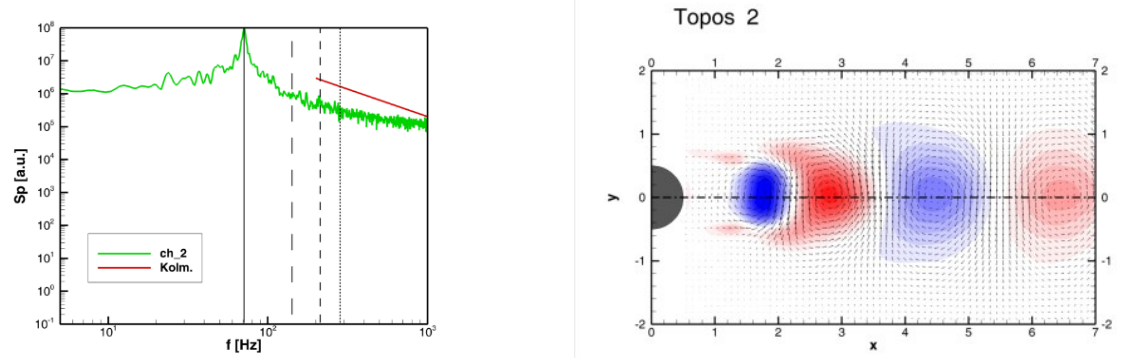

Fig. 9. POD mode 2, spectrum of Chronos (left) and Topos (right).

Group 2: $f_{2}$ and Kolmogorov law.

This group of POD modes is characterized by the peak on fundamental frequency $f_{2}$ and by presence of the Kolmogorov law.

To this group belong the POD modes $3,4,5,6,7,9,10$ and 13. As an example the POD mode 3 is shown in Figure 10 and the mode 9 in Figure 11.
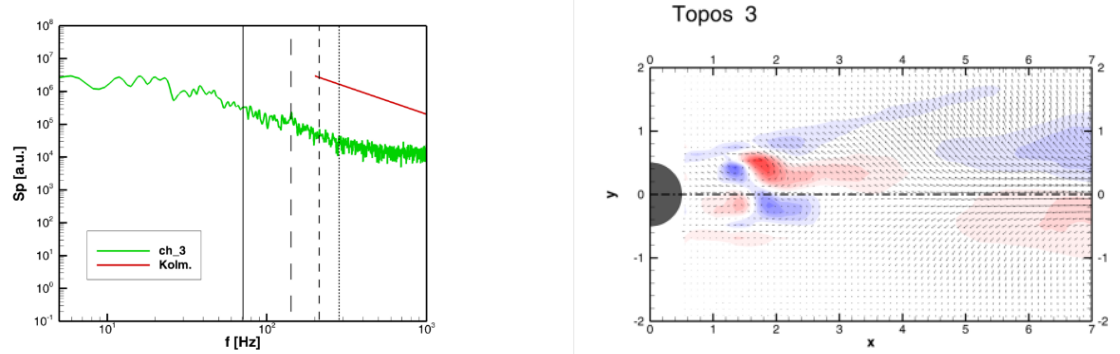

Fig. 10. POD mode 3, spectrum of Chronos (left) and Topos (right). 

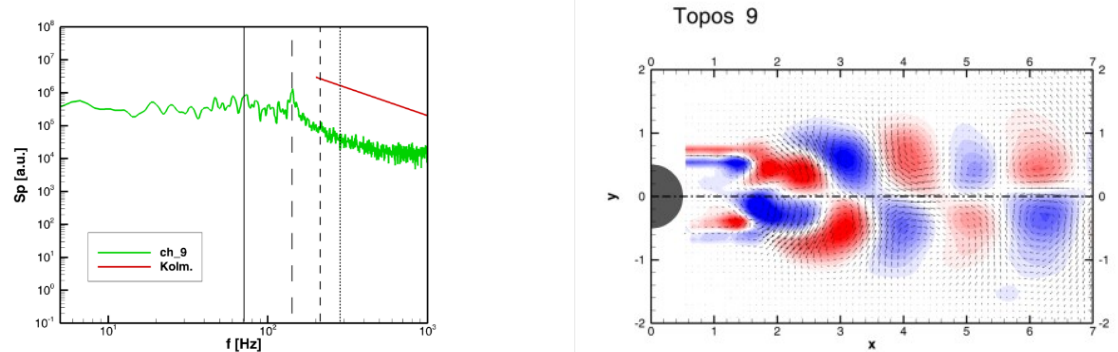

Fig. 11. POD mode 9, spectrum of Chronos (left) and Topos (right).

Group 3: $f_{3}$ and Kolmogorov law.

This group of POD modes is characterized by the peak on fundamental frequency $f_{3}$ and by presence of the Kolmogorov law.

To this group belong the POD modes $8,11,12,14,15,16,17$ and 18. As an example the POD mode 8 is shown in Figure 12 and the mode 15 in Figure 13.
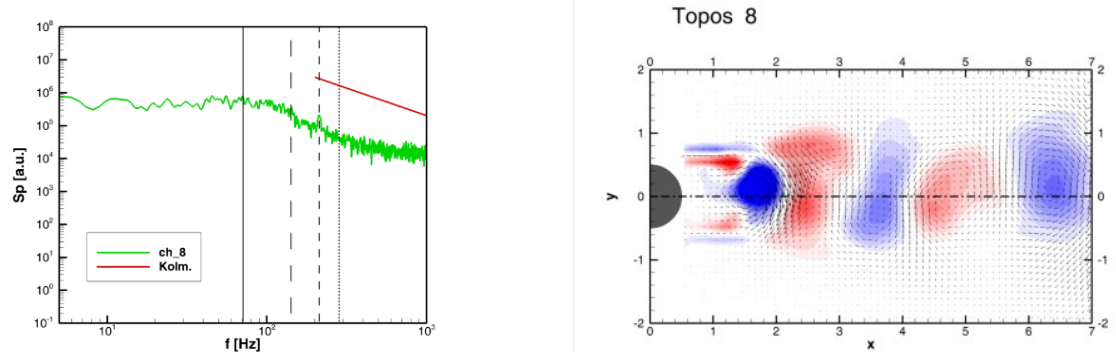

Fig. 12. POD mode 8, spectrum of Chronos (left) and Topos (right).
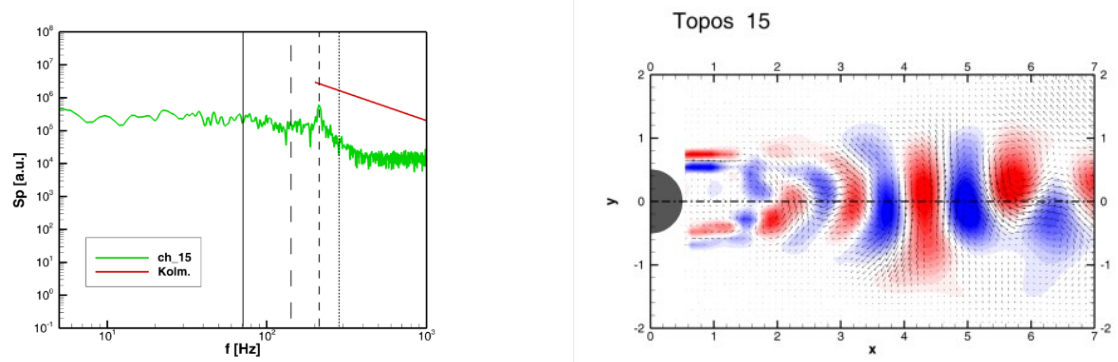

Fig. 13. POD mode 15, spectrum of Chronos (left) and Topos (right).

Group 4: Kolmogorov law.

No peaks only dense spectrum was detected. The high-frequency spectrum shows tendency to the Kolmogorov law, with some part of white noise. The white noise becomes more important with growing mode order.

To this group belong the POD modes of the order between 19 and 500. As an example the POD mode 20 is shown in Figure 14 and the mode 100 in Figure 15. 

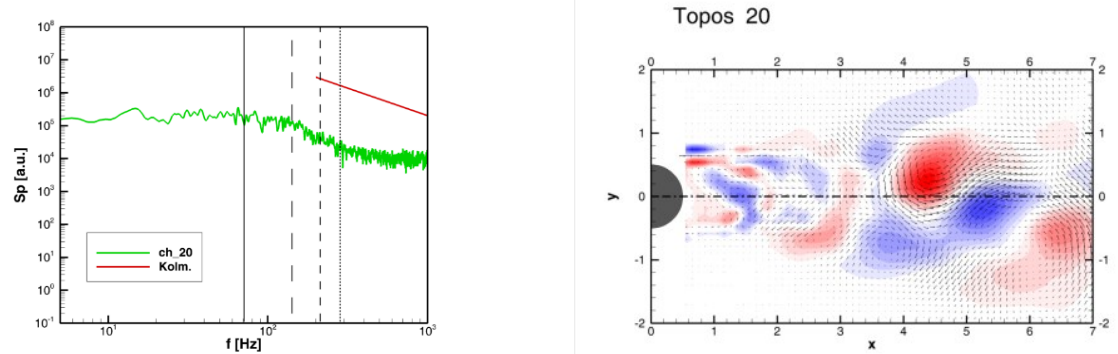

Fig. 14. POD mode 20, spectrum of Chronos (left) and Topos (right).
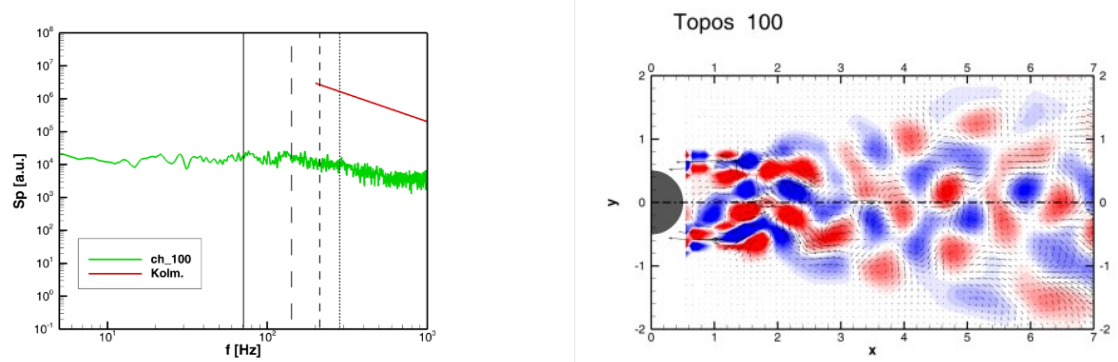

Fig. 15. POD mode 100, spectrum of Chronos (left) and Topos (right).

Group 5: white noise.

In this last group, the dynamical behaviour of the mode is close to white noise characterized by low value of the power spectrum density and constant over the evaluated range of frequencies. The traces of the Kolmogorov law disappeared gradually. Those modes belongs to Gaussian chaotic behaviour, this is demonstrated by the Toposes with chaotic distributions of vectors and vorticity over the whole active region, high values of vorticity indicates high spatial gradients.

To this group belong the POD modes of order higher than approximately 500. As an example the POD mode 500 is shown in Figure 16 and the mode 2000 in Figure 17.
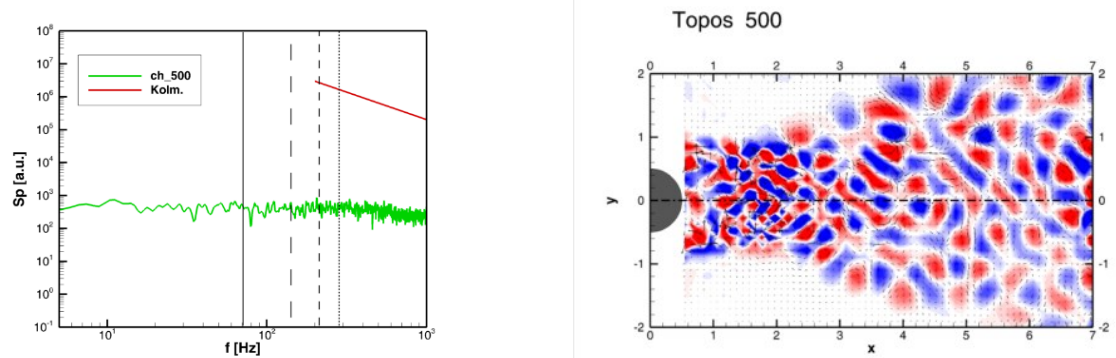

Fig. 16. POD mode 500, spectrum of Chronos (left) and Topos (right). 

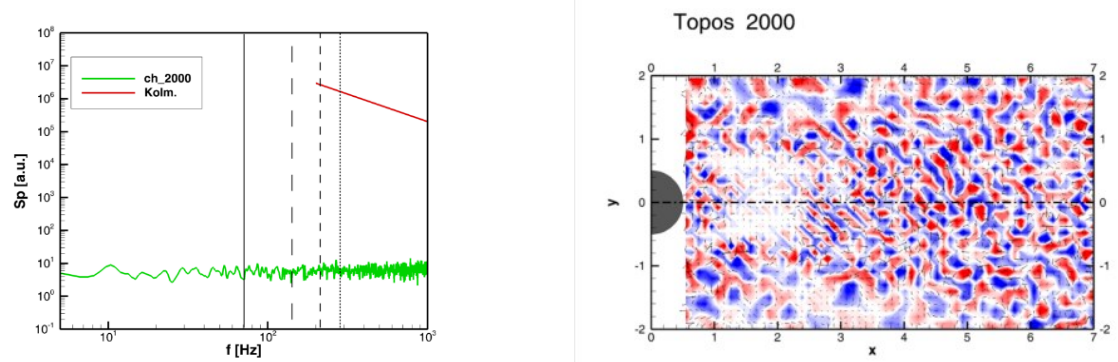

Fig. 17. POD mode 2000, spectrum of Chronos (left) and Topos (right).

Generally, the tendency on the high order modes is to lower frequency and bigger part of the white noise component. The white noise is due to the dynamics generated by measurement uncertainties, including chaotic behaviour of the velocity field and errors of measurement.

The chaotic character of POD modes was studied in [6].

\section{Conclusions}

The wake dynamics behind a long circular cylinder in cross-flow was studied using the POD method.

The temporal parts of POD modes, Chronoses, are subjected to frequency analysis, standard power-spectrum was evaluated. Five groups of modes are distinguished. The first 3 groups of low order modes (up to 19) are characterized by presence of a harmonic content of a given order $\left(1^{\text {st }}, 2^{\text {nd }}\right.$ or $\left.3^{\text {rd }}\right)$ together with a part of spectrum complaining the Kolmogorov law. The higher modes 19-500 contain features, which could be related to the Kolmogorov spectrum in combination with the white noise. The modes of the order higher than 500 contain only white noise and they are supposed to be with predominant random character both in time and space.

The authors acknowledge the financial support of the work by the Grant Agency of the Czech Republic, projects Nos. 17-01088S, 19-04695S and 19-02288J.

\section{References}

1. A. Roshko, On the development of turbulent wakes from vortex streets. NACA Rep. 1191, pp.801-825 (1955)

2. P. Procházka, V. Uruba, Streamwise and spanwise vortical structure merging inside the wake of an inclined flat plate, Mechanics\&Industry, 20, Issue 7, (2019)

3. V. Uruba, On 3D instability of wake behind a cylinder, AIP Conference Proceedings, 1745, (2016), Art. no. 020062

4. V. Uruba, P. Procházka, The Reynolds number effect on dynamics of the wake behind a circular cylinder. AIP Conference Proceedings, 2189, (2019), Article number 020023

5. V. Uruba, Decomposition methods in turbulent research, EPJ Web of Conferences, 25 01095 (2012)

6. V. Uruba, Energy and Entropy in Turbulence Decompositions, ENTROPY, 21, 2, Article Number: 124, (2019), 18pp. 
7. V. Uruba, D. Pavlík, P. Procházka, V. Skála, V. Kopecký, On 3D flow-structures behind an inclined plate, EPJ Web of Conferences, 143, Article number 02137 (2017)

8. C. H. K. Williamson, Vortex Dynamics in the Cylinder Wake, Annu. Rev. Fluid. Mech. (1996) 28, pp 477-539

9. F. M. White, Fluid Mechanics. McGraw Hill, (2015) 